\title{
Possible Role of Lysine Demethylase 2A in the Pathophysiology of Psoriasis
}

\author{
Dong Ha Kim, Mi-Ra Choi, Jae Kyung Lee, Dong-Kyun Hong, Kyung Eun Jung, Chong Won Choi, \\ Young Lee, Chang-Deok Kim, Young-Joon Seo, Jeung-Hoon Lee
}

Department of Dermatology, School of Medicine, Chungnam National University, Daejeon, Korea

Background: Psoriasis is a common chronic inflammatory skin disease. The development of psoriasis is dependent on many intercellular events such as innate immunity and $\mathrm{T}$ cell-mediated inflammation. Furthermore, genetic factors are strongly implicated in the pathophysiology of psoriasis. Although a variety of susceptible genes are identified, it is likely that many important genes remain undisclosed. Objective: The aim of this study is to investigate the possible role of lysine demethylase 2A (KDM2A) in the pathophysiology of psoriasis. Methods: We examined the expression of KDM2A using a well established imiquimod-induced psoriasiform dermatitis model. Results: Immunohistochemistry analysis showed that expression of KDM2A was increased in imiquimod-induced psoriasiform dermatitis. Consistent with this result, KDM2A level was markedly increased in the epidermis of psoriatic patient. When keratinocytes were stimulated with TLR3 agonist poly(I:C), KDM2A was increased at both the mRNA and protein levels. Poly $(\mathrm{I}: \mathrm{C})$ increased the expression of psoriasis-related cytokines including tumor ne-

Received November 27, 2019, Revised March 17, 2020, Accepted for publication June 4, 2020

Corresponding author: Jeung-Hoon Lee, Department of Dermatology, School of Medicine, Chungnam National University, 266 Munhwa-ro, Jung-gu, Daejeon 35015, Korea. Tel: 82-42-280-7707, Fax: 82-42-280-8459, E-mail: jhoon @cnu.ac.kr

ORCID: https://orcid.org/0000-0002-4869-940X

Young-Joon Seo, Department of Dermatology, School of Medicine, Chungnam National University, 266 Munhwa-ro, Jung-gu, Daejeon 35015, Korea. Tel: 82-42-280-7708, Fax: 82-42-280-8459, E-mail: joon@cnu.ac.kr ORCID: https://orcid.org/0000-0002-4955-590X

This is an Open Access article distributed under the terms of the Creative Commons Attribution Non-Commercial License (http://creativecommons. org/licenses/by-nc/4.0) which permits unrestricted non-commercial use, distribution, and reproduction in any medium, provided the original work is properly cited.

Copyright (c) The Korean Dermatological Association and The Korean Society for Investigative Dermatology crosis factor- $\alpha$, interleukin-8, and CCL20, and KDM2A inhibitor daminozide enhanced the poly(l:C)-induced cytokine expression. Finally, topical co-application of imiquimod and daminozide exacerbated the imiquimod-induced psoriasiform dermatitis. Conclusion: Together, these results suggest that KDM2A is increased to negatively regulate the inflammatory reaction of epidermal keratinocytes in psoriasis.

(Ann Dermatol 32(6) 481 486, 2020)

\section{-Keywords-}

Imiquimod, Keratinocytes, Lysine demethylase 2A, Polyinosinic:polycytidylic acid, Psoriasis

\section{INTRODUCTION}

Psoriasis is an inflammatory skin disease that is characterized by papules and silvery white scales, of which condition repeats deterioration and improvement. Although the precise mechanism underlying the onset of disease is not fully understood, it is thought that psoriasis is a T cell-mediated disease and closely related to abnormal keratinocyte differentiation ${ }^{1,2}$. There are lots of immune cell infiltrates in lesional area of psoriasis, and inhibition of $\mathrm{T}$ cells by immunosuppressant ameliorates the disease state ${ }^{3}$. The cytokines produced from $\mathrm{T}$ cells affect epidermal keratinocytes, thereby contributing to development of psoriasis. For example, T cell-derived interleukin (IL)-17A activates epidermal keratinocytes to produce abundant cytokines and inflammatory mediators such as IL-8 and CCL20 ${ }^{4}$. Also, IL-17A inhibits terminal differentiation of keratinoctyes and increases cell proliferation ${ }^{5}$.

Another important factor is genetic background. It has been demonstrated that familial history positively correlates with the development of psoriasis and the probability of occur- 
rence reaches about $70 \%$ between identical twins ${ }^{6}$. Recognizing the importance of genetic background, many investigations identify plenty of susceptible genes using various experimental techniques. For example, linkage analysis identifies distinct disease susceptibility regions (PSORS1-7) ${ }^{7}$. In other example, a genome-wide association study (GWAS) identifies new psoriasis susceptibility loci and an interaction between HLA-C and ERAP ${ }^{8}$. Although a number of genes linked to psoriasis have been discovered, it is likely that many of important genes remain undisclosed. We attempted to find genes related with psoriasis, and found that lysine demethylase 2A (KDM2A) might be involved in the pathogenesis of psoriasis.

KDM2A is a histone 3 lysine 36 (H3K36) demethylase, which plays many roles in biological activities such as cell division, epithelial-mesenchymal transition and cancer cell metastasis ${ }^{9,10}$. KDM2A is known to play an important role in diseases such as cancer, but its role in inflammatory diseases such as psoriasis is not well known. In this study, we suggest that KDM2A is increased to negatively regulate the inflammatory reaction of epidermal keratinocytes.

\section{MATERIALS AND METHODS}

\section{Animal test}

The imiquimod-induced psoriasiform dermatitis model was established according to the method described previously $^{11}$. Male BALB/C mice at 6 to 8 weeks of age were obtained from OrientBio (Seongnam, Korea). The psoriasiform skin inflammation was induced by topical application of $5 \%$ imiquimod cream (Aldara; Dong-A ST, Seoul, Korea) daily for 7 days. All animal experiments were approved by Chungnam National University Institutional Animal Care and Use Committee (CNU-00639).

\section{Immunohistochemistry}

Human skin tissues were obtained with the donors' written consent in accordance with the ethics committee approval procedure of the Chungnam National University Hospital Institutional Review Board (IRB number: 201607-009). Skin tissues were fixed in 10\% formaldehyde and embedded in paraffin. The paraffin-embedded sections were de-waxed, re-hydrated and washed three times with phosphate buffed saline. Sections were incubated with primary antibody for overnight at $4^{\circ} \mathrm{C}$. Sections were then incubated with peroxidase-conjugated secondary antibody (Dako, Carpinteria, CA, USA) and visualized with a Chemmate envision detection kit (Dako). The following primary antibodies were used: KDM2A, CXCL10 (Abcam, Cambridge, MA, USA); CD90 (R\&D Systems, Minneapolis, MN, USA); $\beta$-actin (Sigma-Aldrich, St. Louis, MO, USA).

\section{Cell culture}

The keratinocytes were isolated from the epidermis and immortalized by the transduction of retrovirus harboring Simian virus 40 large T antigen (SV40Tag). Immortalized keratinocytes were maintained in keratinocyte-serum free medium (K-SFM) supplemented with bovine pituitary extract (BPE) and recombinant human epidermal growth factor (rhEGF) (Life Technologies Corporation, Grand Island, NY, USA). When cells reached about $70 \%$ confluency, culture medium was changed with MCDB153 (Welgene, Gyeongsan, Korea) supplemented with BPE and rhEGF. After overnight incubation, cells were treated with $1 \mu \mathrm{g} /$ $\mathrm{ml}$ of poly(I:C) (InvivoGene, San Diego, CA, USA). Daminozide was purchased from Sigma-Aldrich and dissolved in dimethyl sulfoxide (DMSO).

\section{Reverse transcription-polymerase chain reaction (RT-PCR)}

Total RNA was purified and cDNA was prepared using moloney-murine leukaemia virus reverse transcriptase (MMLV-RTase; Elpis Biotech, Daejeon, Korea). Aliquot of CDNA was used in PCR reaction. For quantitative real-time PCR, SYBR Green mixture was used in amplification reaction. The sequence of primers were as follows; KDM2A, 5'-CGGAATTTCTTTTGGGTCAA and 5'-AAGCC TGAGACTGGGCTACA; tumor necrosis factor (TNF)- $\alpha, 5^{\prime}-$ CTCСТTCAGACACCСТCAACCT and 5'-CGACCCTAAGC CCCCAATT; IL-8, 5'-CCTTTCCACCCCAAATTTATCA and 5'-TTTCTGTGTTGGCGCAGTGT; CCL20, 5'-CCACCTCTG CGGCGAAT and 5'-TGTGTATCCAAGACAGCAGTCAAA; GAPDH, 5'-TGCACCACCAACTGCTTAGC and 5'-GGCATG GACTGTGGTCATGAG.

\section{Western blot}

Cellular protein was prepared using a cell lysis buffer (Intron, Daejeon, Korea). Sample was run onto sodium dodecyl sulfate-polyacrylamide gels and transferred onto nitrocellulose membranes (Pall Corporation, Port Washington, NY, USA). After blocking with 5\% skim milk, the membrane was incubated with primary antibody and peroxidase-conjugated secondary antibody sequentially. The Western band was visualized using enhanced chemiluminescence (Intron).

\section{Statistical analysis}

Data were evaluated statistically by one-way ANOVA or Student's t-test using IBM SPSS software ver. 22.0 (IBM Corp., Armonk, NY, USA). Statistical significance was set at $p<0.05$. 


\section{RESULTS}

Imiquimod is an adenosine analogue that belongs to a class of products known as immune response modifiers, and is widely used for the treatment of skin diseases including genital wart, basal cell carcinoma and actinic keratosis $^{12,13}$. Common side effect of imiquimod cream is the provocation of localized psoriasis at the site of application $^{14}$, and this imiquimod-induced psoriasis can be recapitulated in the animal model ${ }^{15}$. We first examined the expression of KDM2A using the imiquimod-induced psoriasiform dermatitis model. Consistent with previous reports, imiquimod induced psoriasiform dermatitis, evidenced by increased epidermal thickness and immune cell infiltrates. The epidermal thickness reached maximum at around 5 day of application, then declined and returned to basal level at 2 weeks. The expression of KDM2A was increased in the epidermis after treatment of imiquimod, which also showed maximum level at around 5 day of application and then declined (Fig. 1).

We next examined the expression of KDM2A in human skin specimens. The expression of KDM2A was weakly detected in the epidermis of normal skin, whereas KDM2A expression was markedly increased in lesional area of psoriatic skin (Fig. 2A). To further investigate the putative involvement of KDM2A, we examined its expression in cultured keratinocytes. We treated keratinocytes with poly(I:C), a synthetic analogue of double-stranded RNA, that induces innate immune response in a TLR3-dependent manner, reflecting the condition of psoriatic keratino-
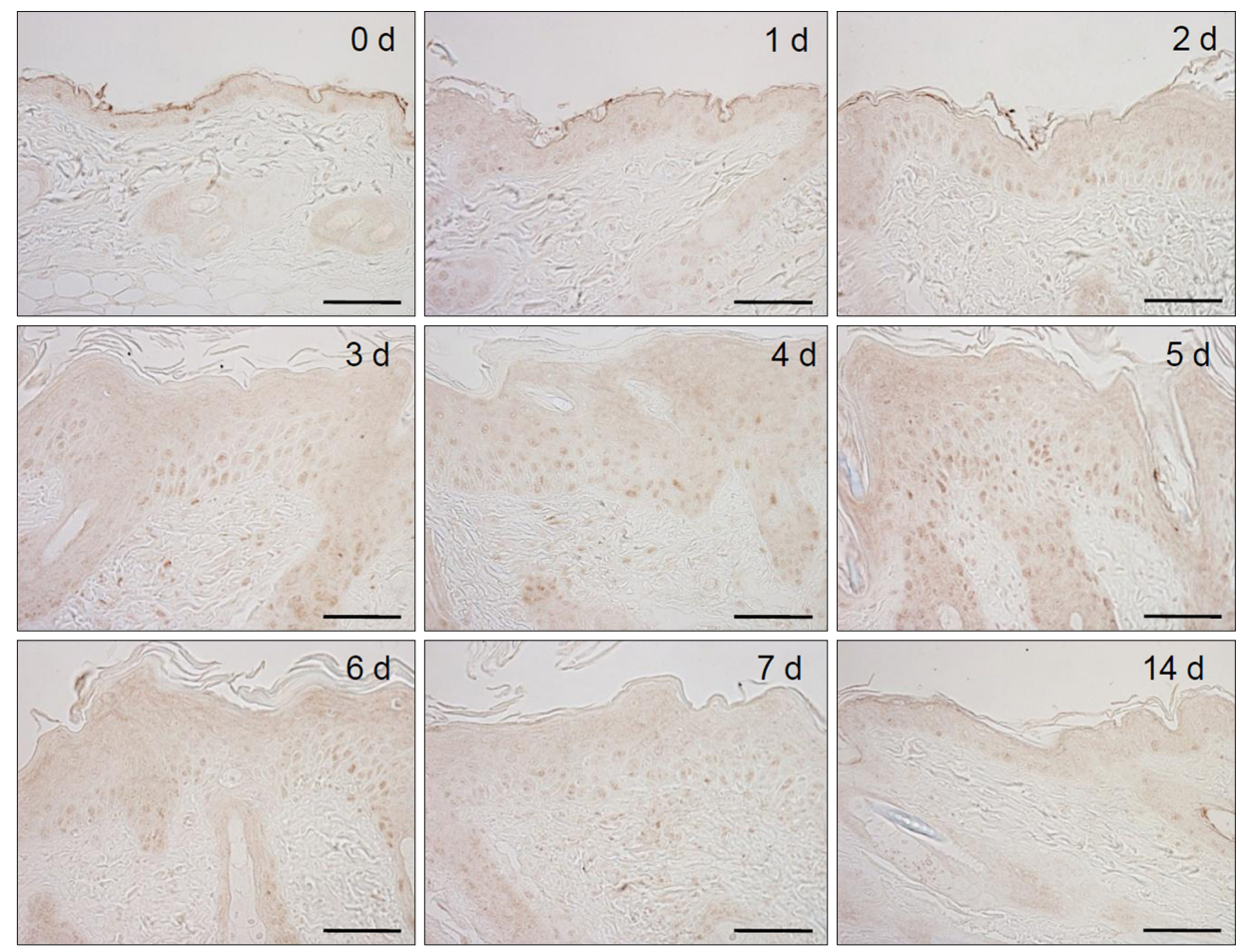

Fig. 1. Expression of lysine demethylase $2 \mathrm{~A}(\mathrm{KDM} 2 \mathrm{~A})$ in the imiquimod-induced psoriasiform dermatitis. BALB/c mice were topically applied with $5 \%$ imiquimod cream (Aldara) daily for 7 days. After final application of imiquimod cream, mice were non-treated for 7 days. Skin specimens were obtained at the indicated time points, and stained with anti-KDM2A antibody. KDM2A level was increased after imiquimod treatment and then declined to basal level in a time-dependent manner. Bar $=100 \mu \mathrm{m}$.
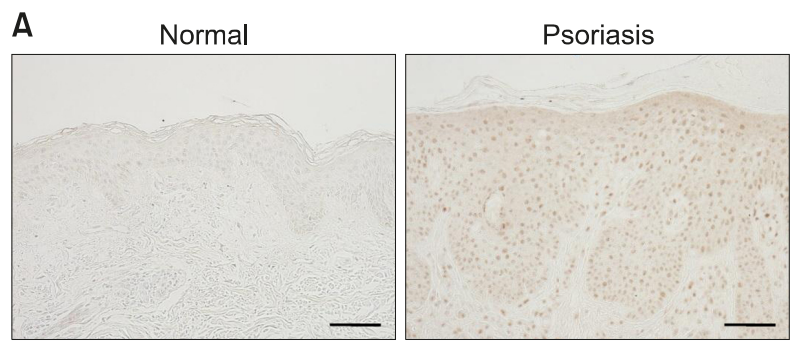

B RT-PCR

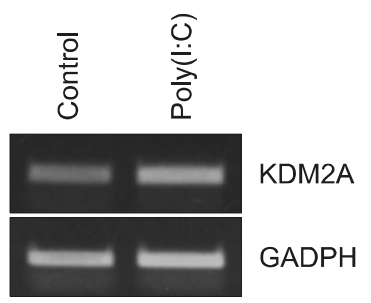

Western

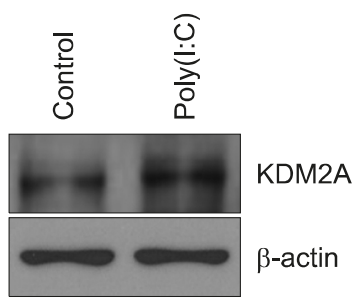

Fig. 2. Expression of KDM2A in human keratinocytes. (A) Skin specimens were obtained from normal volunteer and psoriatic patient. KDM2A level was significantly increased in the epidermis of psoriatic patient. Bar $=100 \mu \mathrm{m}$. (B) Keratinocytes were treated with $1 \mu \mathrm{g} / \mathrm{ml}$ poly(l:C) for 2 hours and then mRNA level was determined by RT-PCR. For Western blot, cells were treated with poly(l:C) for 24 hours and then cellular extract was obtained. Poly $(I: C)$ increased KDM2A at both the mRNA and protein levels. KDM2A: lysine demethylase 2A, RT-PCR: reverse transcription-polymerase chain reaction. 
cytes $^{16}$. Treatment with poly(I:C) led to increase of KDM2A at both the mRNA and protein levels (Fig. 2B). These results suggest that KDM2A may have a role in the patho- physiology of psoriasis.

To investigate the possible role of KDM2A, we treated keratinocytes with KDM2A specific inhibitor daminozide ${ }^{17}$.
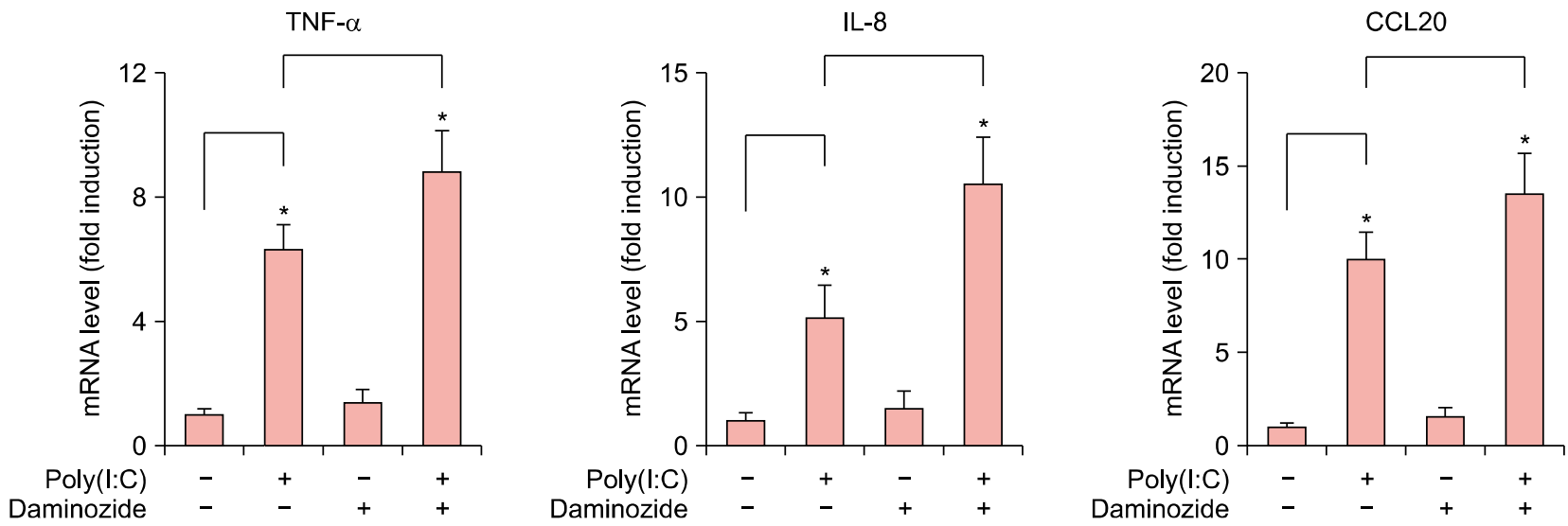

Fig. 3. Effect of lysine demethylase $2 \mathrm{~A}(\mathrm{KDM} 2 \mathrm{~A})$ inhibition on the poly(l:C)-induced inflammatory reaction of keratinocytes. Cells were pretreated with $10 \mu \mathrm{M}$ daminozide (KDM2A inhibitor) for 24 hours, then stimulated with $1 \mu \mathrm{g} / \mathrm{ml}$ poly(l:C) for 2 hours. The mRNA level was determined by quantitative real-time PCR. Data are expressed as fold induction. The mean values \pm standard deviation are averages of triplicate measurements. TNF- $\alpha$ : tumor necrosis factor- $\alpha$, IL-8: interleukin-8. ${ }^{*}$ Statistically significance $(p<0.05)$.

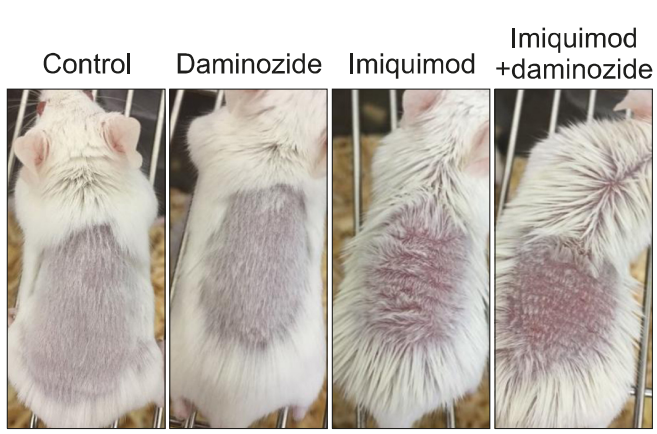

CXCL10

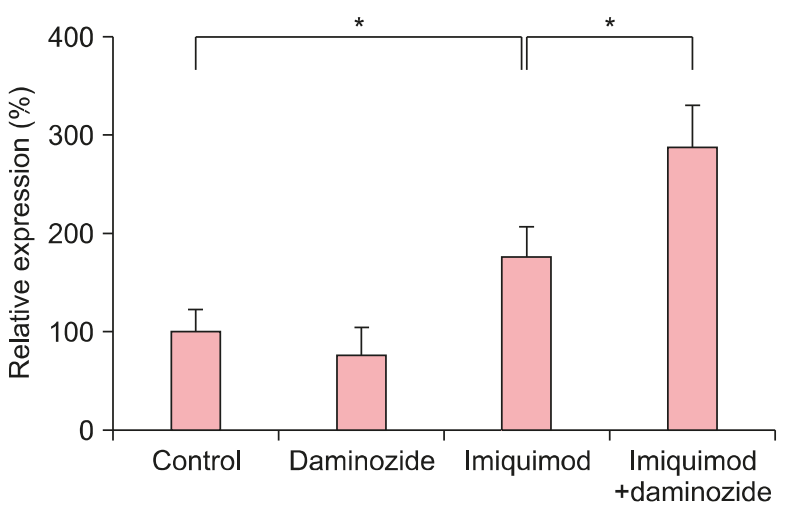

H\&E

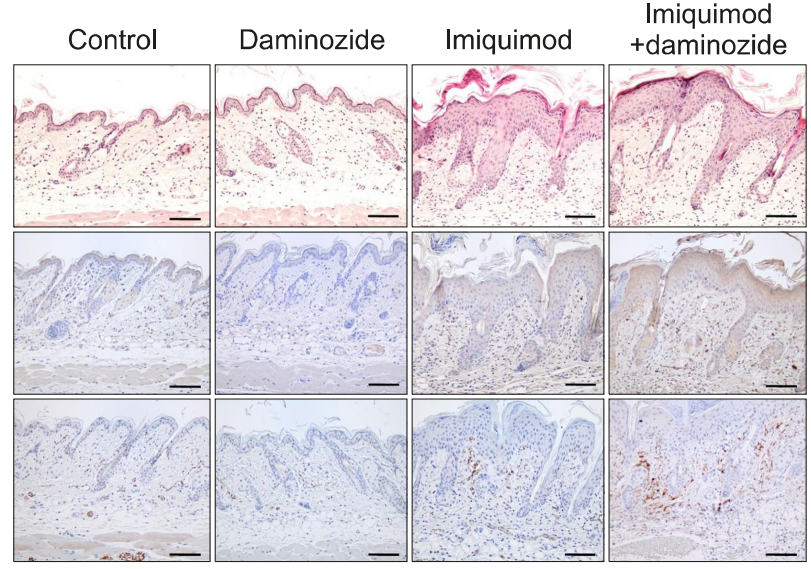

CXCL10

CD90

CD90

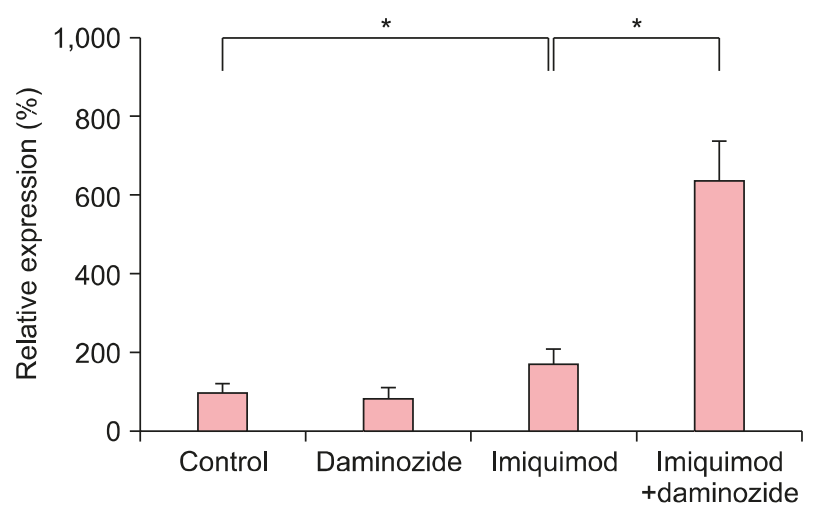

Fig. 4. Effect of lysine demethylase $2 \mathrm{~A}(\mathrm{KDM} 2 \mathrm{~A})$ inhibition on the imiquimod-induced psoriasiform dermatitis. BALB/c mice were coapplied topically with $10 \mu \mathrm{M}$ daminozide and 5\% imiquimod cream daily for 7 days. Skin specimens were obtained 6 hours after final application. Imiquimod increased the epidermal thickness and immune cell infiltration. Co-treatment with imiquimod and daminozide exacerbated the skin inflammation compared to imiquimod only treated-group. Bar $=100 \mu \mathrm{m}$. ${ }^{*}$ Statistically significance $(p<0.05)$. 
Poly (I:C) increased the expression of psoriasis-related cytokines including TNF- $\alpha$, IL-8, and CCL20. When keratinocytes were co-treated with daminozide, cytokine expression was significantly increased compared to poly(l:C) only treated-group (Fig. 3). These results suggest that KDM2A may negatively regulate the inflammatory reaction of epidermal keratinocytes.

We further examined the effect of daminozide using the imiquimod-induced psoriasiform dermatitis model. When daminozide was solely applied, there was no obvious sign of inflammation. By contrast, imiquimod markedly increased the epidermal thickness, together with increase of immune cell infiltrates. Interestingly, co-treatment with imiquimod and daminozide exacerbated the skin inflammation compared to imiquimod only treated-group, in terms of increase of CXCL10 in the epidermis and CD90-positive innate lymphoid cells (Fig. 4). These results support the idea that KDM2A may have a negative role on skin inflammation.

\section{DISCUSSION}

Psoriasis is a complex skin disease, of which occurrence is linked to many susceptible genes. Although a variety of important genes and their functions are identified, it is still unknown how many genes are involved in the pathophysiology of psoriasis. In this study, we demonstrated that KDM2A was increased in psoriasis and it might have a negative role on the inflammatory reaction of keratinocytes.

KDM2A was originally identified as an F-box protein involved in SCF ubiquitin-protein ligase complex ${ }^{18}$. KDM2A contains F-box, JmjC domain and CXXC zinc finger domain, and shows the $\mathrm{H} 3 \mathrm{~K} 36$ demethylase activity ${ }^{19}$. It has been recognized that methylation of H3K36 plays an important role in chromatin remodeling and linked to transcriptionally active state ${ }^{20}$. Thus, it is thought that histone modifier KDM2A is associated with transcriptional silencing. For example, KDM2A represses the transcription of ribosomal RNA (rRNA) in a demethylase activity-dependent manner $^{21}$. In other example, KDM2A transcriptionally represses the histone deacetylase 3 (HDAC3) gene by removing methyl groups from $\mathrm{H} 3 \mathrm{~K} 36$ at the HDAC3 promoter in non-small cell lung cancer (NSCLC) cells ${ }^{22}$. In addition, KDM2A inhibits the expression of tet-eleven translocation 2 (TET2) to promote DNA methylation and silencing of tumor suppressor genes in breast cancer ${ }^{23}$. Since aberrant histone modification is frequently linked to cancer, it is thought that KDM2A plays a role in tumorigenesis. However, the role of KDM2A in inflammatory reaction has not been well elucidated. Interestingly, Lu et al. ${ }^{24}$ have identified $\mathrm{KDM} 2 \mathrm{~A}$ as a negative regulator of NF- $\kappa \mathrm{B}$ using the validation-based insertional mutagenesis. When cells are stimulated with cytokines such as TNF- $\alpha$ and IL- $1 \beta$, the p65 subunit of NF- $\kappa \mathrm{B}$ is methylated at $\mathrm{K} 218$ and K221 positions by lysine methylase NSD1. These methylations are required for the activation of most p65-dependent genes. By contrast, KDM2A demethylates the p65 at K218 and K221 positions, leading to the inhibition of NF$\kappa \mathrm{B}$ activity. Furthermore, expression of $\mathrm{KDM} 2 \mathrm{~A}$ is driven by NF- $\kappa$ B activation, revealing a negative regulatory feedback loop $^{25}$. Since NF- $\kappa \mathrm{B}$ is a central player in inflammation, it can be speculated that KDM2A plays a role in the regulation of inflammatory reaction. In our study, KDM2A was increased in epidermal keratinocytes of psoriatic patient and experimental animal model. Especially in the imiquimod-induced psoriasiform dermatitis model, the expression of $\mathrm{KDM} 2 \mathrm{~A}$ reached maximum level at the highest inflammatory phase, and then declined during the recovery phase. Furthermore, inhibition of KDM2A activity enhanced the poly(l:C)-induced inflammatory reaction of keratinocytes. Finally, topical application of KDM2A inhibitor exacerbated the imiquimod-induced psoriasiform dermatitis. Taken together, these results suggest that KDM2A is increased to prevent the prolonged inflammatory reaction of keratinocytes by suppressing NF- $\kappa$ B activity in psoriasis. Since it has been well described that activation of NF- $\kappa$ B pathway in turn induces the expression of negative regulators for NF- $\kappa \mathrm{B}$ activity, it is suggestive that KDM2A is one of feedback regulators to dampen excessive inflammatory reaction. The precise role of KDM2A in psoriasis should be investigated further.

In summary, we demonstrated that KDM2A was increased in the epidermis of psoriasis, and that inhibition of KDM2A activity exacerbated skin inflammation. Our results suggest that KDM2A may exert its effect as a negative regulator in the inflammatory reaction of keratinocytes.

\section{CONFLICTS OF INTEREST}

The authors have nothing to disclose.

\section{FUNDING SOURCE}

This research was supported by Chungnam National University Hospital Research Fund, 2015.

\section{DATA SHARING STATEMENT}

The data that support the findings of this study are available from the corresponding author upon reasonable request. 


\section{ORCID}

Dong Ha Kim, https://orcid.org/0000-0002-0676-6547

Mi-Ra Choi, https://orcid.org/0000-0003-0734-8360

Jae Kyung Lee, https://orcid.org/0000-0002-9922-5730

Dong-Kyun Hong, https://orcid.org/0000-0002-4244-0691

Kyung Eun Jung, https://orcid.org/0000-0003-0968-1079

Chong Won Choi, https://orcid.org/0000-0001-9994-8819

Young Lee, https://orcid.org/0000-0001-9205-1785

Chang-Deok Kim, https://orcid.org/0000-0001-9341-6491

Young-Joon Seo, https://orcid.org/0000-0002-4955-590X

Jeung-Hoon Lee, https://orcid.org/0000-0002-4869-940X

\section{REFERENCES}

1. Bos JD, de Rie MA, Teunissen MB, Piskin G. Psoriasis: dysregulation of innate immunity. Br J Dermatol 2005;152: 1098-1107.

2. Benhadou F, Mintoff D, Del Marmol V. Psoriasis: keratinocytes or immune cells- which is the trigger? Dermatology 2019;235:91-100.

3. Hawkes JE, Chan TC, Krueger JG. Psoriasis pathogenesis and the development of novel targeted immune therapies. J Allergy Clin Immunol 2017;140:645-653.

4. Brembilla NC, Senra L, Boehncke WH. The IL-17 family of cytokines in psoriasis: IL-17A and beyond. Front Immunol 2018;9:1682.

5. Lai Y, Li D, Li C, Muehleisen B, Radek KA, Park HJ, et al. The antimicrobial protein REG3A regulates keratinocyte proliferation and differentiation after skin injury. Immunity 2012;37:74-84.

6. Chandran V. The genetics of psoriasis and psoriatic arthritis. Clin Rev Allergy Immunol 2013;44:149-156.

7. Semprini S, Capon F, Tacconelli A, Giardina E, Orecchia A, Mingarelli $R$, et al. Evidence for differential S100 gene over-expression in psoriatic patients from genetically heterogeneous pedigrees. Hum Genet 2002;111:310-313.

8. Strange A, Capon F, Spencer CC, Knight J, Weale ME, Allen $\mathrm{MH}$, et al. A genome-wide association study identifies new psoriasis susceptibility loci and an interaction between HLA-C and ERAP1. Nat Genet 2010;42:985-990.

9. Xu WH, Liang DY, Wang Q, Shen J, Liu QH, Peng YB. Knockdown of KDM2A inhibits proliferation associated with TGF- $\beta$ expression in HEK293T cell. Mol Cell Biochem 2019;456:95-104.

10. Lu DH, Yang J, Gao LK, Min J, Tang JM, Hu M, et al. Lysine demethylase $2 \mathrm{~A}$ promotes the progression of ovarian cancer by regulating the PI3K pathway and reversing epithelialmesenchymal transition. Oncol Rep 2019;41:917-927.

11. van der Fits L, Mourits S, Voerman JS, Kant M, Boon L, Laman JD, et al. Imiquimod-induced psoriasis-like skin inflammation in mice is mediated via the IL-23/IL-17 axis. J
Immunol 2009;182:5836-5845.

12. Beutner KR, Tyring SK, Trofatter KF Jr, Douglas JM Jr, Spruance S, Owens ML, et al. Imiquimod, a patient-applied immune-response modifier for treatment of external genital warts. Antimicrob Agents Chemother 1998;42:789-794.

13. Huen AO, Rook AH. Toll receptor agonist therapy of skin cancer and cutaneous T-cell lymphoma. Curr Opin Oncol 2014;26:237-244.

14. Wu JK, Siller G, Strutton G. Psoriasis induced by topical imiquimod. Australas J Dermatol 2004;45:47-50.

15. Madsen M, Pedersen TX, Nielsen LB, Johansen C, Hansen $P R$. Differential effects of digoxin on imiquimod-induced psoriasis-like skin inflammation on the ear and back. Ann Dermatol 2018;30:485-488.

16. Prens EP, Kant M, van Dijk G, van der Wel LI, Mourits S, van der Fits L. IFN-alpha enhances poly-IC responses in human keratinocytes by inducing expression of cytosolic innate RNA receptors: relevance for psoriasis. J Invest Dermatol 2008;128:932-938.

17. Rose NR, Woon EC, Tumber A, Walport LJ, Chowdhury R, $\mathrm{Li} \mathrm{XS}$, et al. Plant growth regulator daminozide is a selective inhibitor of human KDM2/7 histone demethylases. J Med Chem 2012;55:6639-6643.

18. Ilyin GP, Rialland M, Pigeon C, Guguen-Guillouzo C. cDNA cloning and expression analysis of new members of the mammalian F-box protein family. Genomics 2000;67: 40-47.

19. Chen JY, Li CF, Chu PY, Lai YS, Chen $\mathrm{CH}$, Jiang SS, et al. Lysine demethylase $2 \mathrm{~A}$ promotes stemness and angiogenesis of breast cancer by upregulating Jagged1. Oncotarget 2016; 7:27689-27710.

20. Li J, Ahn JH, Wang GG. Understanding histone H3 lysine 36 methylation and its deregulation in disease. Cell Mol Life Sci 2019;76:2899-2916.

21. Tanaka Y, Okamoto K, Teye K, Umata T, Yamagiwa N, Suto $\mathrm{Y}$, et al. JmjC enzyme KDM2A is a regulator of rRNA transcription in response to starvation. EMBO J 2010;29: 1510-1522.

22. Dhar SS, Alam H, Li N, Wagner KW, Chung J, Ahn YW, et al. Transcriptional repression of histone deacetylase 3 by the histone demethylase KDM2A is coupled to tumorigenicity of lung cancer cells. J Biol Chem 2014;289:7483-7496.

23. Chen JY, Luo CW, Lai YS, Wu CC, Hung WC. Lysine demethylase KDM2A inhibits TET2 to promote DNA methylation and silencing of tumor suppressor genes in breast cancer. Oncogenesis 2017;6:e369.

24. Lu T, Jackson MW, Singhi AD, Kandel ES, Yang M, Zhang $Y$, et al. Validation-based insertional mutagenesis identifies lysine demethylase FBXL11 as a negative regulator of NFkappaB. Proc Natl Acad Sci U S A 2009;106:16339-16344.

25. Lu T, Jackson MW, Wang B, Yang $M$, Chance MR, Miyagi $M$, et al. Regulation of NF-kappaB by NSD1/FBXL11-dependent reversible lysine methylation of p65. Proc Natl Acad Sci U S A 2010;107:46-51. 